Journal of Computer Science 8 (1): 121-132, 2012

ISSN 1549-3636

C 2012 Science Publications

\title{
Wireless Body Area Sensor Networks Signal Processing and Communication Framework: Survey on Sensing, Communication Technologies, Delivery and Feedback
}

\author{
${ }^{1,2}$ Khalid Abu Al-Saud, ${ }^{2}$ Massudi Mahmuddin and ${ }^{1}$ Amr Mohamed \\ ${ }^{1}$ Department of Computer Science and Engineering, \\ College of Engineering, Qatar University, Qatar \\ ${ }^{2}$ Awang Had Salleh Graduate School of Arts and Science, \\ University Utara Malaysia, 06010 UUM Sintok, Kedah, Malaysia
}

\begin{abstract}
Problem statement: The Wireless Body Area Sensor Networks (WBASNs) is a wireless network used for communication among sensor nodes operating on or inside the human body in order to monitor vital body parameters and movements. This study surveys the state-of-the-art on Wireless Body Area Networks, discussing the major components of research in this area including physiological sensing and preprocessing, WBASNs communication techniques and data fusion for gathering data from sensors. In addition, data analysis and feedback will be presented including feature extraction, detection and classification of human related phenomena. Approach: Comparative studies of the technologies and techniques used in such systems will be provided in this study, using qualitative comparisons and use case analysis to give insight on potential uses for different techniques. Results and Conclusion: Wireless Sensor Networks (WSNs) technologies are considered as one of the key of the research areas in computer science and healthcare application industries. Sensor supply chain and communication technologies used within the system and power consumption therein, depend largely on the use case and the characteristics of the application. Authors conclude that Life-saving applications and thorough studies and tests should be conducted before WBANs can be widely applied to humans, particularly to address the challenges related to robust techniques for detection and classification to increase the accuracy and hence the confidence of applying such techniques without physician intervention.
\end{abstract}

Key word: Wireless body area sensor network, physiological sensing, data preprocessing, wireless sensor communications, data fusion, classification algorithms, Chronic Disease (CD)

\section{INTRODUCTION}

The worldwide market in 2010 was worth about $\$ 10$ billion for home health monitoring. According to a report from Berg Insight, an analyst firm estimates that the market is growing about 10 percent annually. Berg also mentioned that the market for home health monitoring of chronic diseases was worth about $\$ 11$ billion in 2008. Berg estimates that some 300 million people in the European Union and the United States have at least one or more chronic disease that may benefit from home health monitoring. Eventually, Berg listed cardiovascular irregularities, respiratory problems and diabetes as examples of conditions where home monitoring can become a treatment option. Healthcare industry is one of the world's largest and fastestgrowing industries. Consuming over 10 percent of gross domestic product of most developed countries, healthcare can form an enormous part of a country's economy. Several factors lead to the increasing demand for revolutionary solutions in the healthcare industry, including:

- Increasing number of Chronic Disease (CD) patients; currently more than 860 million; the World Health Organization (WHO) claims. CD accounts for less than $50 \%$ of the population in US and Europe but more than $80 \%$ of the healthcare spending

- Increasing percentage of average of death caused by CDs e.g., $87 \%$ in high income countries

- Percentage of elderly people over 60 is on the rise (WHO, 2010)

Corresponding Author: Khalid Abu Al-Saud, Department of Computer Science and Engineering, College of Engineering, Qatar University, Qatar 
Due to these factors, traditional healthcare cannot provide the scalability required to cope with the growing number of elderly and CD patients as it requires a physical one-to-one relationship between the caregiver and the patient (WHO strategy, 2010). Therefore, the need for high performance, costeffective healthcare solutions is one of the critical strengths for any developing country seeking sustainable future advancements.

A Wireless Sensor Network (WSN) is an infrastructure-fewer network that consists of a number of self-configuring wireless devices capable of sensing vital signs for characterizing contemporary phenomena. Such vital signs include, but not limited to, environmental e.g. air quality, ambient e.g., temperature and pressure and human e.g. heart and brain signals. The sensor data readings are transmitted over a wireless communication channel to a base-station that will be gathering raw data from all sensors, then to a running application that analyzes and makes decisions based on these readings.

Remote monitoring using WSN have recently emerged to provide real-time patient surveillance and to provide CD patients with more autonomy. The conditions most commonly treated by these remote monitoring services include diabetes, cardiac arrhythmia, sleep apnea, asthma and Chronic Obstructive Pulmonary Disease (COPD). Using wireless sensors in the field of healthcare is one of the potential areas, which is expected to save $\$ 25$ billion dollars worldwide by 2012 through leveraging cost-effective solutions and applications as highlighted in the healthcare market report from OnWarld (Chronic disease report from WHO 2011).

WBASNs enable constant monitoring of the health conditions of people with chronicle diseases. WBASN consists of multiple on-body and ambient sensor nodes, capable of sampling, processing and communicating one or more Physiological Signs (PSs) (such as heart activity, brain activity, movements, blood pressure and oxygen saturation) over an extended period. Such physiological signs area measured using different types of sensed signals such as the Electrocardiogram (ECG) (Wikipedia, 2010) Electroencephalogram (EEG)) and acceleration (Wikipedia, 2010). Also, it is used for communications among sensor nodes operating on, or inside the human body in order to monitor vital body parameters and movements as well as to enable its user with quality of life, assisted living, sports, or entertainment purposes (Huang et al., 2009).

In this study, a number of widely applicable capabilities, such as sensing, preprocessing, communication (sending and receiving), data fusion, feature extraction and detection and classifications area briefly discussed. The study focuses on surveying the state-of-the-art on Wireless Body Area Networks (WBASNs), discussing the major components of research in this area including physiological sensing, data preprocessing, data fusion, feature e extraction and detection and classification of human related phenomena. A comprehensive studies and comparisons for sensor technologies used, wireless radio technologies and different detection and classification techniques required to realize the end-to-end WBASNs framework for respiratory-related disorder detection and classification are presented in this study. Our survey framework consists of WBASN which captures medical phenomena that contains the raw data, communication environments, data fusion and major data processing at doctor side.

WBASN signal processing and communication framework: Recent improvements in signal processing and very-low-power wireless communications have motivated great interest in the development and application of wireless technology in healthcare and biomedical research, including Wireless Body Area Sensor Networks (WBASNs). Figure 1 shows a Weans Signal Processing and Communications (WSPC) framework.

WSPC framework consists of three major components for real-time applications, namely Sensing And Preprocessing (SAP), Application-specific WBASN Communication (AWC) and Data Analysis and Feedback (DAF) to the patient. SAP contains a number of sensors for capturing a raw data related to medical phenomena including blood pressure, respiratory rate, ECG and EEG. AWC utilizes application-specific wireless protocols such as ZigBee (Cao et al., 2009) or Bluetooth (Kristina,) to transfer data from body sensors to the gateway, less commonly, in case of high data rates without compression, Wi-Fi protocol may be utilized for intensive data transmission. Analysis of raw data including, possibly, detection and classification of medical anomalies will occur at the DAF component, providing strict and accurate criteria for the physician to make recommendations that maybe sometimes fed back to the patient to provide proactive treatment. Figure 2 shows the conceptual view of the WSPC framework. 


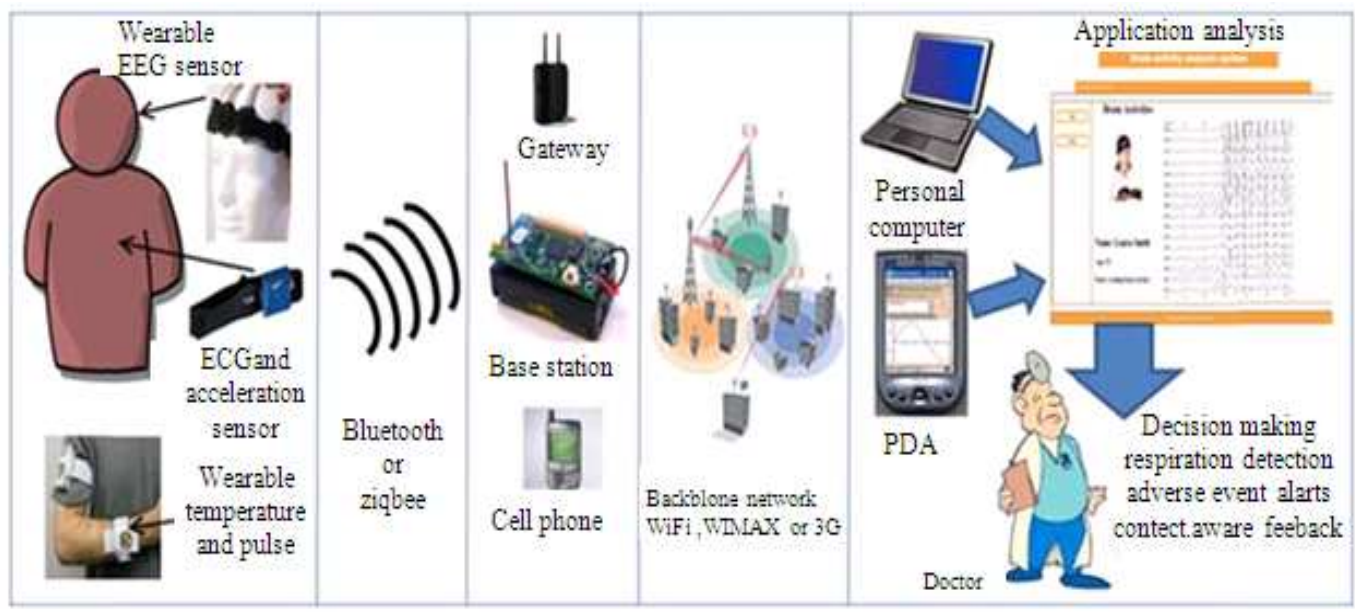

Fig. 1: WBASNs signal process ding and communication framework

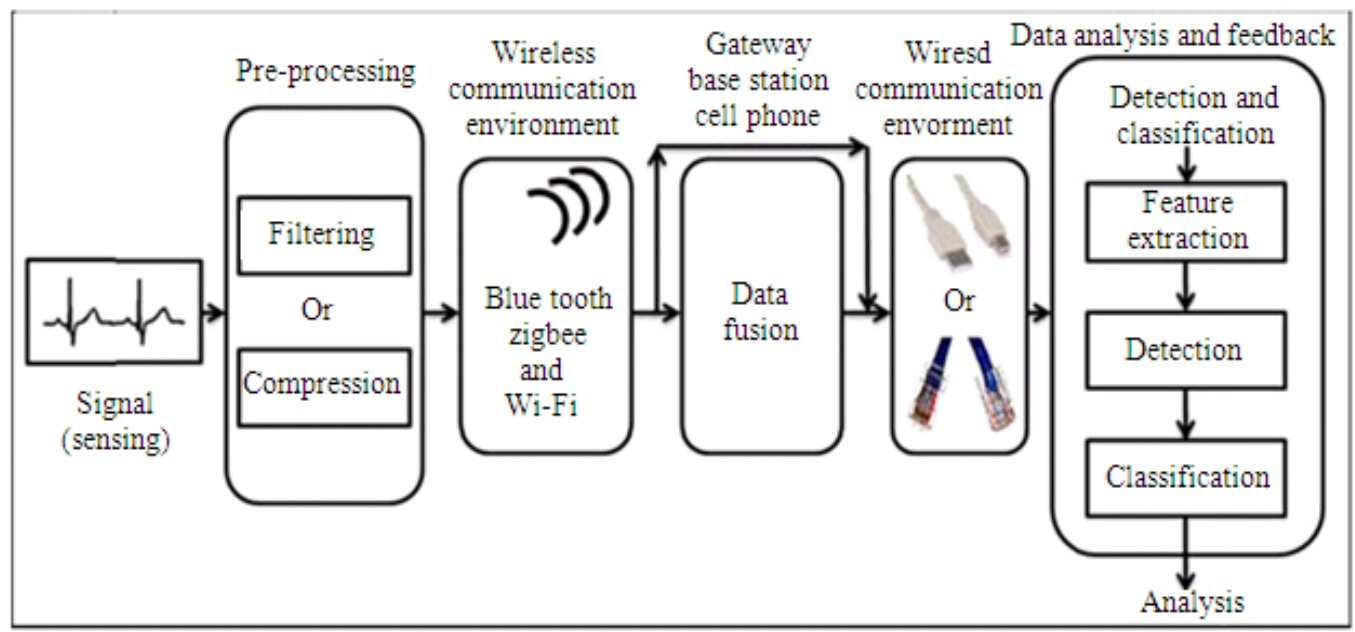

Fig. 2: WSPC conceptual framework

Sensing and preprocessing: Sensor platform architecture typically con sits of a sensing device, an Operating System (OS) and a communication and power management elements. Sensing is the detection of a physical presence of data and the transformation to a signal that can be read by an observer or instrument. A well designed WBAN provides doctors with precise real-time and historical information. It is important that the patient should be comfortable and accepting the system technologies if they are used in daily life.

WBSN can be categorized as single-sensor or multisensor systems. Single-sensor systems use a single unit used on the human body with one channel of communication allocated to it. Multi-sensor systems use multiple units, either of the same or different types of sensors, on patient. The readings from the sensors are usually processed together to extract more accurate readings. In this case, each sensor can have its own communication channel or they can all be combined and synchronized into one channel. The following exact type of sensors used in WBAN that are used in respiration application. It may be single sensor or integrated multi-sensor platform. The sensor can be originated from:

- ECG/EEG/Electromyography (EMG) sensors (for monitoring heart activities, brain activity and skeletal muscles) 
- Pulse Oximetry (monitoring amount of oxygen that is being "carried" in a patient's blood)

- Blood glucose level sensor in the patient's

- Body temperature sensor (Ullah et al., 2009)

To better observe a human's vitals signals, a wide range of commercial sensor technologies are used to capture physical data such as, accelerometer, ECG electrodes, EEG electrodes, EMG, pulse oximetry, respiration rate, heart rate, blood pressure, blood sugar and temperature sensors will be deployed. ECG and EEG electrodes are manufactured in several types, including disposable electrodes, reusable electrodes, headbands or caps and needle electrodes. For example, an ECG electrode is a device a attached to the skin on certain parts of the patient's body such as arms, legs and chest to detect electrical impulses produced each time the heart beats. Electrode position for a 12-lead ECG is standard, with leads placed on both the left and right arm and leg. On contrast, EEG neuron-feedback is gathered by placing electrodes on approximately 20 different areas of the scalp (http://www.wisegeek.com/topics/eeg-electrodes.htm\#). The EEG electrodes are placed according to the International 10-20 system. EEG generally uses six "exploring" electrodes and two "reference" electrodes. Another example is the EMG, which typically uses four electrodes to measure muscle tension as well as to monitor for an excessive amount of leg movements during sleep (http://en.wikipedia.org/wiki/Polysomnography). Table 1 show sensors employed in WBANs systems and their typical data rate.

The wireless sensor node OS plays a fundamental role in the overall capabilities and performance of the platform. Early research into OS for sensor networks lead to the development of TinyOS by researchers at. OS is used in the processing of signals captured. A transceiver communication unit allows the transmission and reception of data to other devices that connecting a wireless sensor node to a network. Details about the communication environment will be discussed. Power management provided by the operating system to enforce an optimal way of utilizes energy. Conserving power involves accessing/controlling components on the sensor node. The components which expose power management interfaces are processor, radio and battery. The components that can be controlled to conserve power are processor and radio. Increasing number of electrodes affects the mobility and convenience of the human subject. The more electrodes we use, the more accurate the data will be. However, this will affect the mobility of the user negatively.
Preprocessing manifested by the procedures performed on the raw data to be ready for analyzes and processing by the application. Preprocessing technique transforms the data into a format that will be more easily and effectively processed for the user purpose (http://searchsqlserver.techtarget.com/definition/datapreprocessing). Kotsiantis et al. (2006) address issues of data pre-processing that can have a significant impact on performance of the data analysis, including feature extraction and detection, which will be covered. Data preprocessing includes data filtering, compression, etc. They present a well-known algorithm for each step of data preprocessing in case if there is much irrelevant and redundant information present or noisy and unreliable data. Xu (2009) presented a model of the data preprocessing to reduce the energy consumption attribute to communication between the nodes and enhance the effectiveness of data transmission of wireless sensor networks by means of utilizing independent and intelligent multi-agents. Also, the model presents the algorithm to accomplish data preprocessing and avoids the error of the data collection. Also, Ahmad et al. (2009) present a software application that resides in the Personal Digital Assistant (PDA) carried by each patient. This application periodically performs an initial assessment of the patient's condition based on the available data to work reducing the level of data. If the parameter is within the normal range of health, the input data for that period will be decreased to a few representative values only. Otherwise, all the data will be transferred over the wireless network to the central database for further analysis. However, before transmitting the digitized data and as part of preprocessing inside the sensor, two major procedures may be performed, namely data filtering and data compression.

Data Filtering: Filtering refers to the process of defining, detecting and correcting errors in sensed data, due to mixing with external noisy data, with the purpose of minimizing the impact of these errors for the succeeding analyses. Filter scans the data for multiples/clones/duplicates of records and inconsistent data to exclude it or taking the average of some measured values over a certain time period. Whenever unnecessary data occur, one representative record is chosen or constructed. The rest are deleted and the data is reduced in size as a result. The output will finally be a refined data, but still potentially contain errors. An effective energy-saving filtering mechanism is proposed in (Huang and Huixu, 2010) to enhance the energy-efficiency of data-gathering. 
Table 1: Description of common sensors in WBAN systems (Cao et al., 2009; Bourbakis and Pantelopoulos, 2010)

\begin{tabular}{|c|c|c|c|c|}
\hline Sensor type & Signal type & Description of measured data & Compression needed & Sample rate \\
\hline Accelerometer & Body Move & Measure the three dimensional acceleration & Required for 3D & $100 \mathrm{KHz}$ \\
\hline ECG/EEG/ & Skin/ scalp & Measure Electrical activity of the heart, brain & Recommend & $250 / 250$ \\
\hline EMG & Electrodes & activity and skeletal muscles respectively & & $2 \mathrm{khz}$ \\
\hline Pulse Oximetry & $\begin{array}{l}\text { Oxygen } \\
\text { Saturation }\end{array}$ & $\begin{array}{l}\text { Measure the oxygenation or the amount of } \\
\text { oxygen that is being "carried" in a patient's blood }\end{array}$ & Not required & $<1 \mathrm{~Hz}$ \\
\hline Heart rate & Pulse ox meter/ & Frequency the cardiac cycle & Not required & $60 \mathrm{khz}$ \\
\hline Blood pressure & $\begin{array}{l}\text { Arm cuff } \\
\text { Based monitor }\end{array}$ & Measures the systolic pressure and diastolic pressure & Not required & $<1 \mathrm{~Hz}$ \\
\hline Blood glucose & $\begin{array}{l}\text { Strip-base } \\
\text { glucose meters }\end{array}$ & $\begin{array}{l}\text { Measurement of the amount of glucose (main } \\
\text { type/source of sugar/energy) in blood. }\end{array}$ & Not required & $<1 \mathrm{~Hz}$ \\
\hline $\begin{array}{l}\text { Temperature } \\
\text { Probe }\end{array}$ & $\begin{array}{l}\text { Body and/or } \\
\text { skin temperature }\end{array}$ & $\begin{array}{l}\text { A measure of the body's ability to generate and } \\
\text { get free of heat }\end{array}$ & Not required & $<1 \mathrm{~Hz}$ \\
\hline
\end{tabular}

The proposed filtering framework mainly puts emphasis on reducing the production of redundant loads at the gathering source to greatly reduce energy cost using self-adaptive filtering scheme, which is constructed by prediction module for modifying model and driving module for executing filtering operation. The goal of filtering mechanism is to achieve the energy-saving effect by reducing the redundant communication loads in networks, which is the tradeoff between the data precision and energy-efficiency. Finally, it is concluded that the proposed filtering mechanism can do better than some classical data gathering approaches on the aspect of energy-saving effect. To reduce the sensor data volume that arises from the use of continually-transmitting sensors (e.g., ECG or EMG), Homed et al. (2008) proposed a context-aware filtering technique in which the relaying mobile device dynamically modifies its processing logic based on changes in the user's context. They implemented the Healthcare-oriented Adaptive Remote Monitoring (HARMONI) middleware in order to evaluate this technique, on a mobile device and used it to collect real sensor data from users. HARMONI includes a lightweight event engine that runs on the mobile device and processes incoming sensor data streams using rules that are appropriate for the current context. Their experiments demonstrate that context-aware filtering can reduce the uplink bandwidth requirements of the system by up to $72 \%$.

Compression: In contrast to filtering, which potentially focuses on reducing errors and unnecessary data (such as duplicate data), compression focuses on reducing the amount of physical data traffic that the sensor sends over the wireless channel, to improve bandwidth utilization, power consumption and possibly speed of processing and memory space required by the application. Data compression can be categorized into two methods: lossless and loss. Lossless method promotes the reconstruction of the original signal after compression with no loss of any type. While preserving all the signal characteristics, this method may require excessive bandwidth for communication. Also, depending on the application, signals may not be required in full to be able to detect the patient's anomalies. In contrast, loss method will not get the original signal accurately after compression but may be more bandwidth efficient, while minimizing the effect on the application processing. Lossy method used two major criterions: The Compression Ratio (CR) that is representing the ratio between the original signal and compressed signal; the percentage root-mean-square difference (PRD) which defined as the error criterion in estimating of signal rebuilt for lossy compression (Hua et al., 2010). The error criterion for lossy compression techniques estimate the distortion of the signal rebuilt with respect to the original one is very important, especially for ECG signal, where a slight loss or change of information can lead to wrong diagnostics. The controlled transmission quality measure PRD for ECG compression is described in Eq. 1 as:

$$
P R D=\sqrt{\frac{\sum_{1-I}^{N}(x(i)-\hat{x}(i) 2}{\sum_{1-I}^{N}(x(i)-\mu) 2}} \times 100 \%
$$

Where:

$$
\begin{array}{ll}
\mathrm{x}(\mathrm{i}) \text { and } \mathrm{x}(\mathrm{i}) & =\mathrm{i}^{\text {th }} \quad \text { samples of original and } \\
& \text { reconstructed ECG signals of length } \\
\mathrm{N} \text { and } \mu & =\begin{array}{l}
\text { signal mean value }
\end{array}
\end{array}
$$

Kim et al. (2010) proposed quad level vector for ECG signal processing to achieve a better performance for both compression flow and classification flow with lowcomputation complexity. The classification algorithm was employed for the heartbeat segmentation and the R-peak detection methods. The overall energy consumption cost is reduced by $45.3 \%$ with the proposed compression techniques. Vidhyapriya and Vanathi (2009) design and implement two lossless data compression algorithms namely entropy encoded codebook compression and pipelined codebook compression which are basically built over the codebook compression techniques. 
They assumed each sensor node is stationary after deployment and is capable of getting its location information using GPS through camera sensor network. The advantages of this technique are the reduced amount of memory occupied by the compression and the short processing time required. This leads to a reduction of the overall delay of the data packet transmission. Pradhan et al. (2002) proposed a framework for distributed compression using joint source and channel coding. Their approach can be extended to capture more elaborated correlation structures.

\section{WBASNS communication technologies:}

Communication technologies in WBASN are a radiofrequency wireless networking technology-based that interconnects tiny nodes through sensor or actuator capabilities in, or inside a human body. WBASN healthcare applications use different types of wireless protocols like Bluetooth (IEEE 802.15.1) and Zigbee (IEEE 802.1.5.4 Standard) as well as Wi-Fi (based on 802.11 b) with different change of each technology for low power and long range. Each WBASN consists of multiple of interconnected nodes on, or within a human body, which together provide a sensing, processing and communication efficiencies. A WBASN system has its own characteristics, as listed in Table 2.

A brief description on Bluetooth technology (based on IEEE 802.15.1 protocol), ZigBee technology (based on IEEE 802.1.5.4 standard protocol) and Wi-Fi as sensor communication environment is reviewed. Wi-Fi based on $802.11 \mathrm{~b}$ protocol, in case if there is a high data rate and no compression, is discussed as well.

Bluetooth: Bluetooth is a wireless technology designed to connect different wireless devices such as telephones, notebooks, PDAs, printers, computers and so on. It is also low power and low processing with an overhead the protocol, which is means, the applications with Bluetooth are virtually endless. Bluetooth isn't line of sight and it provides short-range (30 feet) of $10 \mathrm{~m}$, which can be increased up to $100 \mathrm{~m}$, Bluetooth operates in the $2.4 \mathrm{GHz}$ band with moderately fast transmission speeds (data rate) of $800 \mathrm{~Kb} \mathrm{sec}^{-1}$ (Krasteva, 2005). Unlike legacy MAC technology with low throughput e.g., (Ganapathy and Sagayaraj, 2010), Bluetooth offers only $1 \mathrm{MBps}$ and its security is low due to the greater range and Radio Frequency (RF) that make it more open to interception and attack. Eventually, Bluetooth as shortrange wireless technology is very suitable for many medical applications. The Bluetooth Health Device Profile (HDP) (Latuske and Software GmbH, 2009) was released by the Bluetooth special interest group in June 2008, which contributed to the propagation of Bluetooth in many healthcare applications. Bluetooth and Wi-Fi use the same frequency range, but employ different modulation techniques.

Zig bee: Zig Bee is a standard targeting low-datatransfer-rate, low-power-consumption and low-cost wireless applications. Zig Bee is used for wireless control and sensing inside a home or hospital if the application does not talking to a phone McDonald, (2011). It lower physical data rate is traded for lower power consumption and together with a simpler protocol stack, Zig Bee devices enjoy relatively longer lifetime. It uses a wide range of frequencies: sixteen channels in the $2.4 \mathrm{GHz}$. It can accommodate up to $2^{64}$ nodes in the network (Stankovic et al., 2005). It is corresponding to Bluetooth technology in terms of infrastructure-oriented mesh networking support (Cao et al., 2009). It applications are different from home and industrial automation to remote control and medical monitoring. Ken and Xiaoying (2010) used Zig bee in design and implement an intelligent system for remote monitoring ECG, analysis and diagnosis. Zig Bee is designed if a number of different radios were deployed in a particular area, the network will figure our automatically without user intervention taking care of retries, in addition to, the network self-recovery, acknowledgements and routing data message. The technology found in Zig Bee is intended to be simpler and expensive than Bluetooth.

Wireless Fidelity (Wi-Fi): Wi-Fi refers to certain types of wireless network protocol $802.11 \mathrm{~b}$ standards to communicate devices to each other without cords or cables. It is like a conventional Ethernet network, needs configuration to set up shared resources, transmit files and to set up audio links (hands-free devices and headsets). It uses the same radio frequencies as Bluetooth, but with higher power, resulting in a stronger connection. Wi-Fi is more convenient for running fullscale networks; it enables better range from the base station, a faster connection and better security than Bluetooth. Wi-Fi generally uses super ultra-low noise Sband $(2.4 \mathrm{GHz})$ to eliminate cable loss and to extend the range and frequency-hopping techniques to connect multiple devices together, has a range of about 1000 feet outdoors. It is mainly intended to be used for LAN in residential homes, for public access hotspots and in business. 
Table 2: WBASN system characteristic

\begin{tabular}{|c|c|}
\hline Characteristic & Description \\
\hline Architecture & $\begin{array}{l}\text { WBASNs have certain sensor nodes on or inside a human body; but in Wireless Personal } \\
\text { Area Network (WPAN) includes router node around human body as an infrastructure for } \\
\text { sending data away from WBASNs. Every node is a sensor node as well as a router node }\end{array}$ \\
\hline Density & $\begin{array}{l}\text { The number of sensor nodes deployed on/in body depends on the application; sensor nodes } \\
\text { grouped into different groups, but routers is widely distributed }\end{array}$ \\
\hline Data rate & $\begin{array}{l}\text { Most WSNs are applied for remote monitoring, where events can occur irregularly. In } \\
\text { comparison, human's physiological activities are mostly periodic and as a result, the } \\
\text { generated packet streams have steady data rates }\end{array}$ \\
\hline Latency & $\begin{array}{l}\text { For certain medical applications, latency caused by underlying communications network of } \\
\text { a WBASN-WPAN system is critical. Power saving is definitely useful in WBASN-WPAN, } \\
\text { but certain nodes could be always on rather than go to sleep often and whenever necessary } \\
\text { to change the battery }\end{array}$ \\
\hline Mobility & $\begin{array}{l}\text { Humans move. Even those people with particular medical treatment are likely to move } \\
\text { sometimes. Compared to equipments wired bedside which limit patients' mobility, WBASN } \\
\text { takes benefit of wireless connections. However, this also contributes to the complexity of } \\
\text { the network (Cao et al., 2009) }\end{array}$ \\
\hline
\end{tabular}

Table 3: Comparison between Zig Bee, Bluetooth and Wi-Fi

\begin{tabular}{llll}
\hline Category & ZigBee & Bluetooth & Wi-Fi \\
\hline Cost & Cheap & Cheap & Inexpensive \\
Ease of use & Not Easy & Easy & Acceptable \\
Range & 20-25 feet & 30 feet & 100 feet \\
Reliability & Reliable & Not very reliable & Not very reliable \\
Flexibility & Good & Poor & Fair \\
Scalability & Excellent & Poor & Fair \\
Power Consumption & Low & Average & High \\
Interoperability & Good & Excellent & Excellent \\
\hline
\end{tabular}

This technology is found in computers and PDA allowing Internet through access points called hotpots. Therefore Wi-Fi can be also used for online communication method to notify and alerts doctor if both the doctor and patient are in one building (Zhao and Zhan, 2005). Despite the high power consumptions of Wi-Fi-based devices this technology will become essential for intensive health signals with high data rates and lightweight or no compression. The main application of Bluetooth is to replace cables in a variety of small-scale applications, whereas Wi-Fi is used to provide wireless high speed connectivity for general local area network access. Wi-Fi differs from Bluetooth in that it covers greater distances and provides higher throughput, but requires more expensive hardware and may present higher power consumption. The following Table 3 shows the comparison between ZigBee, Bluetooth and Wi-Fi (Krasteva, 2005; Khan et al., 2009).

Gateway data fusion: Data fusion is commonly known as the use of technique(s) that can combine both data and related information from multiple sensors in order to achieve correct and reliable data. This will be more efficient and potentially accurate when compared with the use of a single sensor. Data fusion is typically performed by a reference sensor node for aggregating and making inferences about the gathered data. It deals with the combination of multiple sources by mixing data together to obtain enhanced data which is cheaper, higher quality or better relevance. Within the WSNs domain, simple aggregation techniques such as maximum, minimum and average, have been developed for reducing the overall data traffic to save energy. Data fusion techniques, however, can reduce not only the amount of data traffic, but also filter noisy measurements and make expectations and conclusions about the monitoring entity (Nakamura et al., 2007). The personal server or mote implemented on a PDA, cell phone, gateway, or personal computer, coordinates the data collection from sensor nodes using a TimeDivision Multiple Access (TDMA) mechanism and transfers data to the central server. Physicians can access data on the central server via the internet and warning signals can be created by an agent running on the server. However, the power consumption and cost associated with long-term data uploading can hinder system achievement (Cao et al., 2009). In recent years, data fusion has been growing rapidly as the requirements on the accuracy of nondestructive testing and evaluation are increasing in the Non-Destructive Evaluation (NDE) community. Multiple NDE methods are often used to increase the reliability and reduce the uncertainty of the test and evaluation. Authors Liu et al. (2007) give an overview in NDE data fusion techniques and examine the mathematical fusion algorithms, which motivates solution to the explanation of NDE data. They classify NDE data fusion into two types: feature integration and feature classification or characterization. In addition, the research in (Wang et al., 2008) proves that the realization of data fusion algorithm in application layer can improve power and network lifetime, which eventually can also enlarge the range of application of ZigBee protocol. Since ZigBee protocol includes physical layer, MAC layer, network layer and application layer (Stojmenovi, 2005). 
Physical layer and MAC layer are realized by RF chip, so data fusion can only be combined with network layer and application layer. Doing data fusion comes down to semantic gap between data fusion and application data. Data fusion based on application data can realize maximum data compression. However, it will be hard to realize protocol because of the question followed by understanding of spanning layers when data fusion combines with network layer. To realize real time data fusion and multivariate signal processing on sensor level, Liu et al. (2007) developed a slim network layer protocol for sensor-driven autonomous real-time data fusion in a star WBASN. To adapt sensor network to changing external conditions the protocol allows reconfiguration of the sensors during measurements being initiated by sensor nodes themselves, the network coordinator, or an external expert.

Data analysis and feedback: One of the important healthcare areas are concerning with the respiration system, which is one of the most important systems for human to survive. The primary function of the respiratory system is to supply the blood with oxygen in order for the blood to deliver oxygen to all the body. The rate of respiration plays a key role in intensive care and neonatal. The most common way to monitor the rate of respiratory has been the visual observation of the patient, but in recent times has involved modern technologies in this area. Hundreds of millions of people worldwide suffer every day from chronic respiratory diseases. According to the WHO estimates in 2007, currently $300 \mathrm{~m}$ people have asthma; 210 million people have COPD, while millions have allergic rhinitis and other often under-diagnosed chronic respiratory diseases (WHO, 2010). In order to help automate the process of dealing with these types of diseases and due to large volume of acquired data required to diagnose them, sophisticated techniques are required for analysis to extract relevant features, which can help in efficiently detecting and classifying anomalies with high precision. In this part, data analysis and feedback at the server side will be discussed, which includes feature extraction, detection and classification.

Feature extraction: ECG Feature Extraction plays a significant role in diagnosing most of the cardiac diseases. Recently, numerous research and techniques have been developed for analyzing the ECG signal. The proposed schemes were mostly based on fuzzy logic, artificial neural networks, support vector machines and other signal analysis techniques. Zhao and Zhan (2005) proposed a feature extraction method using wavelet transform and support vector machines. The study presented a new approach to the feature extraction for reliable heart rhythm recognition. The wavelet transform is used to extract the coefficients of the transform as the features of each ECG segment. Concurrently, Autoregressive Modeling (AM) is also applied to get hold of the temporal structures of ECG waveforms. The results of show that a high level of accuracy for the classification and recognition of 6 heart rhythm types of up to $99.68 \%$ can be obtained. The features extracted will then be used to detect an adverse patient event using a function of all the features measured, without classifying the severity of this event. On the other hand, classification is used to provide grade levels for the severity of the patient's adverse event. The research work (Noh, 2007) tries to find out significant Heart Rate Variability (HRV) signal through comparison between power spectrums of ECG-Derived Respiration (EDR) and R-R interval variability ratio. The result shows that by considering cross-correlation, which is the measure of similarity of both HRV and EDR signals as a function of a time-lag applied to one of them, significant data acquisition gain can be achieved, while disregarding the low frequency component representing the Respiration Sinus Arrhythmia to enhance the feature extraction quality before classification. Karlen et al. (2009) proposed a method for the estimation of the homeostatic component with a wearable device, where sleepiness increasing over daytime cause of accidents in transportation and factory plants. The method uses a Fast Fourier Transform as the main feature extraction tool and a feed-forward artificial neural network as a classifier. However, the proposed methods produces a more balanced correct classification of sleep and wake periods. Additionally, by adjusting the classification threshold of the neural classifier, $86.7 \%$ of correct classification is obtained as a result. Tadejko and Rakowski (2007) proposed a mathematical morphology for ECG feature extraction. The primary focus of their work is to evaluate the classification performance of an automatic classifier of the ECG for the detection abnormal beats with new concept of feature extraction stage. The obtained feature sets were based on ECG morphology and RR-intervals. (Tadejko and Rakowski, 2007) evaluates various combinations of morphological filters and performs experiments for different structuring elements. Their preliminary results show that the proposed algorithm leads to an improvement in the classification of heart rate depends on shape and size of structuring element. Since the opening and closing operations are prepared to remove impulses, the structuring element must be designed whenever that the waves in the ECG signal are not removed by a process. 
Detection and classification: ECG detection and classification is essential to the timely diagnosis and analyze potentially fatal and chronic diseases proactively in clinical as well as various life settings. There are two categories representing the performance of classification which are specificity and sensitivity, which are defined as a function of the true and false positives and negatives. First, False Positives (FP) refers to the condition in which the results are perceived as positive, when there is no definite disease or severe illness. On the other hand, True Positives (TP) is a test that shows correct behavior by detecting definite disease or severe illness. Similarly, we can define True Negatives (TN) as the correct behavior to detect normal patient condition with no severe illness, while False Negatives (FN) are the incorrect detection of normal condition, where the subject suffers a severe disease or illness.

Specificity in diagnostic laboratory refers to the ability of an assessor to measure one particular organism or substance (Hua et al., 2010). Specificity is a medical term defined as a percentage ratio of true negative tests to the total number of unaffected patients tested. Moreover, sensitivity in diagnostic laboratory testing represents the smallest amount of substance in a sample that can be accurately measured by an assessor. Sensitivity is defined as a percentage ratio of true positive tests to the total number of affected (positive) patients tested. Therefore, specificity and sensitivity can be defined as follows:

$$
\begin{aligned}
& \text { Specificity }=\frac{\mathrm{TN}}{\mathrm{TN}+\mathrm{FP}} \\
& \text { Spensitivity }=\frac{\mathrm{TP}}{\mathrm{TP}+\mathrm{FN}}
\end{aligned}
$$

These Eq. 2 and 3 show that a test with high specificity has few FPs, where as a test with high sensitivity has few FNs.

Bsoul et al. (2010) have developed a low-cost, realtime sleep apnea monitoring system "Apnea MedAssist" for recognizing Obstructive Sleep Apnea (OSA) period with a high degree of accuracy for both home and clinical care applications. The system uses patient's single-channel ECG to extract feature sets and uses Support Vector Classifier (SVC) to detect apnea incident and to achieve a classification F-measure of $90 \%$ and a sensitivity of $96 \%$ for the subjectindependent SVC. The real-time capability comes from the use of 1 minute segments of ECG periods for feature extraction and classification. Automatic detection and classification of cardiac arrhythmias is important for diagnosis of cardiac abnormalities. Chronic obstructive pulmonary disease is a major public health problem. Patel et al. (2009) present a comparative analysis of classification performance of a set of different classification techniques and factors that affect classification performance for activity recognition based on accelerometer data. Also, perform simulations to determine an appropriate value of window length to extract features and the features that are most informative. The result was low classification error; better classification performance can be achieved with careful selection of sensors and the use of reduced sensor set. Prasad and Sahambi (2003) proposed a method to accurately classify ECG arrhythmias through a combination of wavelet transforms and artificial neural networks. The ability of the wavelet transform to decompose signal at various resolutions allows accurate extraction/detection of features from non-stationary signals like ECG. A set of discrete wavelet transform coefficients, which contain the maximum information about the arrhythmia, is selected from the wavelet decomposition. These coefficients are fed to the backpropagation neural network which classifies the arrhythmias. The proposed method is capable of distinguishing the normal sinus rhythm and 12 different arrhythmias and is robust against noise. The overall accuracy of classification of their proposed approach is 96.77\%. The ECG beat signal variations in both shape and time imposes great challenges to automatic detection tasks. To address those challenges and accurate automatic detection, the research study $\mathrm{Li}$ (2010) proposed a new ECG beat classifier based on both time and transform domain characters. The result shows that this method overcomes the locality of classification performance and achieves high detection ratio on large dataset, which goes a big step further towards the commercial application. Gomathi and Thangaraj (2010), designed a hardware Smart Electronic Nose System (SENS) based on neural networks to classify different adsorbed odors. Okubo et al. (2008) proposed an automatic nebulizer control a new home-care system based on continuous respiration monitoring for such patients. They employed a masktype unit has a capacitance microphone to take care of an elderly person at home and detect the sound of cough to install a measurement sensor for respiration together with a nebulizer. Okubo et al. (2008) confirmed that the proposed system could measure respiratory rate accurately, detect the change of respiratory condition and the cough. Jan et al. (2008) presented a study that aims at detecting obstructive and restrictive pulmonary abnormalities using a combined approach of leveraging artificial neural networks with 
the data gathered from sprirometer. Spiro metric data was then statistically analyzed concerning its significance for neural networks. They obtained the data from 250 adult volunteers with standard recording protocol in order to detect and classify normal and abnormal pulmonary diseases. The results show that the proposed method could be useful for detecting the function of respiratory system.

Delivery and feedback: Delivery and feedback provides useful channels to deliver the information resulting from the data analysis to relevant stockholders to help enhance treatment and provide proactive measures to both patients and physicians. Data analysis will lead to useful feedback to the patient; we can summarize them in three categories-namely information, recommendation and diagnostic. The information feedback (Durham et al., 2009) focuses on sending transparently, to the patient, the information output from the detection and classification phase, without any data inference that can lead to diagnosing the patient symptom. The physician will then look at the analyzed data to provide the appropriate treatment to the patient and send the appropriate feedback to do so. The recommendation feedback focuses on automatic extraction of possible treatments for the patient and presents this to the physician merely as a recommendation. The physician will look at the recommendations and decide what treatment will be provided to the patient as a feedback. Lastly, diagnostic feedback is in a way a futuristic concept, where the physician can be removed from the loop for certain illnesses. In this scenario, the information outcome from the data analysis phase can be used to search in a medical treatment database using some intelligent techniques to provide the appropriate treatment for the patient without the physician intervention.

\section{CONCLUSION}

In this study we have provided a survey of this promising field through a survey of pioneer WBASNs research projects and enabling technologies, including, sensing and preprocessing, communication environments of WBANs, data analysis and feedback that have feature extraction, detections and classifications. In particular, for life-saving applications, thorough studies and tests should be conducted before WBANs can be widely applied to humans. Compression is used to reduce the amount of physical data traffic that the sensor send in a small size to improve and utilize bandwidth communication, power consumption and memory space. Sensor supply chain and communication technologies used within the system depend largely on the use case and the characteristics of the application. On other hand, the feature extraction, detection and classification, play a vital role in diagnosing most of the cardiac diseases and they provide efficient tools for enhancing the diagnosis of illnesses in various clinical and life settings.

\section{ACKNOWLEDGMENT}

The researchers would like to acknowledge the support of Qatar University. This research study is part of project number: NPRP 09 -310-1-058, funded by Qatar National Research Fund (QNRF) http://www.qnrf.org/, State of Qatar.

\section{REFERENCES}

Ahmad, N.F., D.B. Hoang and M.H. Phung, 2009. Robust preprocessing for health care monitoring framework. Proceeding of the 11th IEEE International Conference on e-Health Networking, Applications and Services, Dec. 16-18, IEEE Xploor, Sydney, pp: 169-174. DOI: 10.1109/HEALTH.2009.5406196

Bourbakis, N.G. and A. Pantelopoulos, 2010. A survey on wearable sensor-based systems for health monitoring and prognosis. IEEE Trans. Syst., Man Cybernetics-Part C: Appli. Rev., 40: 1-12. DOI:10.1109/TSMCC.2009.2032660

Bsoul, M., H. Minn and L. Tamil, 2010. Apnea medassist: Real-time sleep apnea monitor using single-lead ECG. IEEE Trans. Inform. Technol. Biomed., 15: 416-427. DOI: 10.1109/TITB.2010.2087386

Cao, H., V. Leung, C. Chow and H. Chan, 2009. Enabling technologies for wireless body area networks: A survey and outlook. IEEE Commun. Mage., 47: 84-93. DOI: 10.1109/MCOM.2009.5350373

Cao, H., X. Liang, I. Balasingham and V.C.M. Leung, 2009. Performance analysis of ZigBee technology for wireless body area sensor networks. Lec. Notes Institute Comput. Sci. Soc. Inform. Telecommun. Eng., 28: 747-761. DOI: 10.1007/978-3-642-117237_51

Durham, K., P.M. Van Vliet, F. Badger and C. Sackley, 2009. Use of information feedback and attentional focus of feedback in treating the person with a hemiplegic arm. Physiotherpy Res. Int., 14: 77-90. DOI: $10.1002 /$ pri.431 
Ganapathy, G. and S. Sagayaraj, 2010. Circumventing picture archiving and communication systems server with hadoop framework in health care services. J. Soc. Sci., 6: 310-314. DOI: 10.3844/jssp.2010.310.314

Gomathi, M. and P. Thangaraj, 2010. A computer aided diagnosis system for lung cancer detectionlusing support vector machine. Am. J. Applied Sci., 7: 1532-1538. DOI: 10.3844/ajassp.2010.1532.1538

Homed, I., A. Misra, M. Ebling and W. Jerome, 2008. HARMONI: Context-aware filtering of sensor data for continuous remote health monitoring. Proceedings of the 6th Annual IEEE International Conference on Pervasive Computing and Communications, Mar. 17-21, IEEE Xploor, Hong Kong, pp: 248-251. DOI: 10.1109/PERCOM.2008.110

Hua, K., H. Wang, W. Wang and S. Wu, 2010. Adaptive data compression in wireless body sensor networks. Proceedings of the 13th IEEE International Conference on Computational Science and Engineering, Dec. 11-13, IEEE Xploor, Hong Kong, pp: 1-5. DOI: 10.1109/CSE.2010.65

Huang, L., M. Ashouei, F. Yaziciogl, J. Penders and R. Vullers et al., 2009. Ultra-low power sensor design for wireless body area networks: Challenges, potential solutions and applications. Int. J. Digit. Cont. Technol. Applied, 3: 1-36. DOI: 10.4156/jdcta.vol3.issue3.17

Huang, R. and G. Huixu, 2010. The design of energysaving filtering mechanism for sensor networks. Proceedings of the 9th International Conference on Machine Learning and Cybernetics, Qingdao, July 2010, IEEE Xploor, Qingdao, pp: 79-85. DOI: 10.1109/ICMLC.2010.5581088

Karlen, W., C.o. Mattiussi and D. Floreano, 2009. Sleep and wake classification with ECG and respiratory effort signals. IEEE Trans. Biomed. Circ. $\quad$ Syst., 3: 71-78. $\quad$ DOI: 10.1109/TBCAS.2008.2008817

Ken, C. and L. Xiaoying, 2010. A zigbee based mesh network for ECG monitoring system. Proceedings of the 4th International Conference on Bioinformatics and Biomedical Engineering, June 18-20, IEEE Xploor, Chengdu, pp: 1-4. DOI: 10.1109/ICBBE.2010.5514693

Khan, P., M.A. Hussain and K.S. Kwak, 2009. Medical applications of wireless body area networks. Interna. J. Digi. Cont. Technol. Appl., 3: 3. DOI: 10.4156/jdcta.vol3.issue3.23

Kim, H., R.F. Yazicioglu, P. Merken, C. Van Hoof and H.J. Yoo, 2010. ECG Signal compression and classification algorithm with quad level vector for ECG holter system. IEEE Trans. Inform. Technol.
Biomed., $\quad$ 14: $\quad 93-100 . \quad$ DOI: 10.1109/TITB.2009.2031638

Kotsiantis, S.B., D. Kanellopoulos and P.E. Pintelas, 2006. Data preprocessing for supervised leaning. Int. J. Compt. Sci., 1: 111-117.

Krasteva, R., A. Boneva, V. Georchev, I. Stoianov, 2005. Application of wireless protocols bluetooth and ZigBee in telemetry system development. central laboratory of mechatronics and instrumentation. Bulgarian Acad. Sci., 55: 30-38.

Latuske, R., A.R.S. Software GmbH, 2009. Bluetooth health device profile and the IEEE 11073 medical device frame work. Software GmbH.

Li, A., S. Wang, H. Zheng, L. Ji and J. Wu, 2010. A novel abnormal ECG beats detection method. Proceedings of the 2nd International Conference on Computer and Automation Engineering, Feb. 26-28, IEEE Xploor, Singapore, pp: 47-51. DOI: 10.1109/ICCAE.2010.5452002

Liu, Z., D.S. Forsyth, J.P. Komorowski, K. Hanasaki and T. Kirubarajan, 2007. Survey: State of the art in NDE data fusion techniques. IEEE Trans. Instrument. Measure., 56: 6-6. DOI: 10.1109/TIM.2007.908139

McDonald, M.P., 2011. Enterprises don't need social media, they need to become a social organization. Gartner, Inc.

Nakamura, E.F., A. Loureiro and A. Frery, 2007. Information fusion for wireless sensor networks: Methods, models and classifications. ACM Computing Surveys (CSUR), 39: 3. DOI: 10.1145/1267070/1267073

Noh, Y., S.B. S. Park, K. Hong and H. Yoon, 2007. A study of significant data classification between EDR extracted and frequency analysis of heart rate variability from ECG using conductive textile. World Cong. Med. Phys. Biomed. Eng., 14: 41004103. DOI: 10.1007/978-3-540-36841-0_1039

Okubo, M., Y. Imai, T. Ishikawa, T. Hayasaka, S. Ueno and T. Yamaguchi, 2008. Development of automatic respiration monitoring for home-care patients of respiratory diseases with therapeutic aids. Int. Federation Med. Biolog. Eng., 22: 11171120, DOI: 10.1007/978-3-540-89208-3_267

Patel, S., C. Mancinelli, J. Healey and M. Moy, 2009. Using wearable sensors to monitor physical activities of patients with COPD: A comparison of classifier performance. Proceeding of the 6th International Workshop on Wearable and Implantable Body Sensor Networks, June 3-5, IEEE Xploor, Berkeley, pp: 234-239. DOI: 10.1109/BSN.2009.53 
Pradhan, S.S., J. Kusuma and K. Ramchandran, 2002. Distributed compression in a dense microsensor network. IEEE Signal Proc. Mag., 9: 51-60. DOI: $10.1109 / 79.985684$

Prasad, G.K. and J.S. Sahambi, 2003. Classification of ECG arrhythmias using multi-resolution analysis and neural networks. Proceeding of the Conference on Convergent Technologies for Asia-Pacific Region, Oct. 15-17, IEEE Xploor, Asia, pp: 227231. DOI: 10.1109/TENCON.2003.1273320

Stankovic, J.A., Q. Cao, T. Doan, L. Fang and Z. He et al., 2005. Wireless sensor networks for in-home healthcare: Potential and challenges. University of Virginia.

Stojmenovi, I., 2005. Handbook of Sensor Networks: Algorithms and Architectures University of Ottawa. John Wiley Sons Inc. 2005, ISBN: 978-047168472-5.

Tadejko, P. and W. Rakowski, 2007. Mathematical morphology based ECG feature extraction for the purpose of heartbeat classification. Proceeding of the 6th International Conference on Computer Information Systems and Industrial Management Applications, June 28-30, IEEE Xploor, Minneapolis, pp: 322-327. DOI: 10.1109/CISIM.2007.47

Ullah, S., P. Khan, N. Ullah, S. Saleem and H. Higgins, 2009. A review of wireless body area networks for medical applications. Int. J. Commun. Network Syst. Sci., 2: pp: 797-803. DOI: 10.4236/ijens.2009.28093
Vidhyapriya, R. and P. Vanathi, 2009. Energy efficient data compression in wireless sensor networks. Int. Arab. J. Inform. Technol., 6: 297-303.

Wang, P., M.C. Cheng and W.Z. Shi, 2008. Research on data fusion algorithm in ZigBee protocol. Proceeding of the International Symposium on Computer Science and Computational Technology, Dec. 20-22, IEEE Xploor, Shanghai, pp: 524-527. DOI: 10.1109/ISCSCT.2008.339

WHO, 2010. Country Cooperation Strategy (CCS) 2008-2011. World Health Organization.

$\mathrm{Xu}$, M., 2009. Research and design of data preprocessing of wireless sensor networks based on multi-agents. Proceeding of IEEE International Conference on Network Infrastructure and Digital Content, IC-NIDC Nov. 6-8, IEEE Xploor, Beijing, China, pp: 50-53. DOI: 10.1109/ICNIDC.2009.536073

Zhao, Q. and L. Zhan, 2005. ECG feature extraction and classification using wavelet transform and support vector machines. Proceeding of the International Conference on Neural Networks and Brain, Oct. 13-15, IEEE Xploor, Beijing, pp: 10891092. DOI: 10.1109/ICNNB.2005.1614807

Zhao, Y., Y. Yagi, H. Juzoji and I. Nakajima, 2005. A study of Wireless IP for Telemedicine. Tokai University. 